\title{
Rat Models of Autoimmune Uveitis
}

\author{
Gerhild Wildner Maria Diedrichs-Möhring Stephan R. Thurau \\ Section of Immunobiology, Department of Ophthalmology, Medical Center of the University of Munich, \\ Ludwig-Maximilians-University, Munich, Germany
}

\section{Key Words}

S-antigen - Interphotoreceptor retinoid-binding protein •

Peptide $\cdot$ T lymphocyte $\cdot$ Antigenic mimicry $\cdot$ Intravital

fluorescence microscopy $\cdot$ Oral tolerance

\begin{abstract}
Experimental autoimmune uveitis (EAU) in Lewis rats is a well-established model for human uveitis. During the last years we used this model to demonstrate extraocular induction of uveitis by antigenic mimicry of environmental antigens with retinal autoantigen and investigated the migration and intraocular reactivation of autoreactive green fluorescent protein (GFP)+ T cells. We could also elaborate several differences between EAU induced with S-antigen peptide PDSAg or R14, a peptide derived from interphotoreceptor retinoid-binding protein, suggesting two differently regulated diseases in the same rat strain. R14-mediated EAU in Lewis rats has been shown to relapse, thus we have a new model to test therapeutic approaches in an ongoing immune response instead of just preventing disease. Finally, we show antigenic mimicry of PDSAg and an HLA-B peptide for oral tolerance induction. After the successful first therapeutic trial this approach will now proceed with international multicenter clinical trials.

Copyright $\odot 2008$ S. Karger AG, Basel
\end{abstract}

(C) 2008 S. Karger AG, Basel

0030-3747/08/0404-0141\$24.50/0

Fax +4161306 1234

E-Mail karger@karger.ch

www.karger.com
Accessible online at: www.karger.com/ore

\section{Autoantigens of Rat Experimental Autoimmune Uveitis}

Experimental autoimmune uveitis (EAU) in Lewis rats is an intraocular inflammation leading to retinal destruction. The disease is mediated by CD4+ T helper 1 (Th1) cells, which recruit inflammatory cells, mainly monocytes/macrophages. In the rat eye, both the anterior and the posterior parts are affected, whereas irreversible destruction is mainly observed in the retina. EAU can be induced by immunization with several retinal autoantigens, of which retinal S-antigen (S-Ag) and interphotoreceptor retinoid-binding protein (IRBP) are best characterized. The most pathogenic epitopes of these autoantigens cover amino acids (AA) 341-354 of S-Ag (peptide PDSAg) [1] and AA 1169-1191 of IRBP (R14) [2].

\section{Peripheral Induction of a Cross-Reactive Immune Response by Antigenic Mimicry}

The immune privilege of the eye, maintained by the blood-retina barrier, anterior-chamber-associated immune deviation (ACAID) and immunosuppressive mediators (i.e. TGF- $\beta$ or $\alpha$-melanocyte-stimulating hormone $(\alpha-\mathrm{MSH})$ ), prevents ocular invasion of nonactivated leucocytes. Therefore, an autoimmune response

Gerhild Wildner, PhD, Section of Immunobiology

Department of Ophthalmology, Medical Center of the University of Munich

Mathildenstrasse 8, DE-80336 Munich (Germany)

Tel. +49895160 3888, Fax +498951603045

E-Mail Gerhild.Wildner@med.uni-muenchen.de 
leading to intraocular inflammation has to be generated outside of the eye, and we postulate a cross-reactive nonocular antigen as the activator of $\mathrm{T}$ cells (antigenic mimicry). $\mathrm{T}$ cells activated to such a mimotope are then able to enter the eye because activated $\mathrm{T}$ cells can pass the blood-retina barrier. In the eye they are reactivated by cross-reactive retinal antigen. There is one exception to this mechanism of disease induction, which is sympathetic ophthalmia. Here, the injury to one eye allows the entry of immune cells to ocular tissue and a direct generation of an ocular antigen-specific immune response.

We could describe two mimotopes of retinal peptide PDSAg, peptides derived from environmental antigens. One peptide, Cas, covers AA 73-84 of bovine milk $\alpha$ s2casein and is a typical nutritional antigen, the other, Rota, is derived from the outer capsid protein (AA 591-601) from gastroenteritis-causing rotavirus [3]. Both peptides induce uveitis after immunization with complete Freund's adjuvant (CFA), with a lower incidence but the same disease severity as with PDSAg. T cell lines specific for Rota and Cas are also pathogenic; moreover, they show crossreactivity to PDSAg in vitro. Not only Cas peptide but also $\alpha$ s2-casein protein is able to induce uveitis in Lewis rats. The disease is indistinguishable from PDSAg-induced EAU with respect to clinical course and histology. Since nutritional antigens are normally applied to the gastrointestinal mucosa and not subcutaneously, we also tried to induce EAU by feeding the antigens together with native cholera toxin, which is a Th1-shifting gastrointestinal adjuvant in rats. Only casein protein, but none of the peptides or S-Ag was able to induce EAU by the oral route, indicating that a gastrointestinal immune response to cross-reactive antigens might be a way to induce ocular autoimmune disease. Uveitis patients showed significantly higher immune responses ( $\mathrm{T}$ cells, antibodies) to retinal S-Ag, peptide and the mimotopes than healthy controls.

\section{Intraocular Reactivation of Cross-Reactive T Cells}

To follow the migration of autoreactive $\mathrm{T}$ cells, we injected antigen-activated, green-fluorescent-protein (GFP)-transduced R14- or ovalbumin (OVA)-specific control T cells intravenously into naive rats. The migration of fluorescent $T$ cells was observed by daily intravital fluorescence microscopy of the iris [4]. The first GFP+ T cells, irrespective of their specificity for autoantigen or OVA, extravasated and entered the iris tissue within 30 min after injection. The number of GFP+ cells of either specificity gradually increased within the first 3 days. After 3 days the number of OVA-specific GFP+ T cells decreased, whereas R14-specific T cells increased, accompanied by an infiltration of GFP-inflammatory cells. At this time point the clinical disease started, with ophthalmoscopically visible infiltration of leucocytes. The time between the immigration of the first autoreactive $\mathrm{T}$ cells and the recruitment of inflammatory cells is probably the prodromal state, which patients with anterior uveitis sometimes experience as an 'organ feeling' or pain preceding a clinically visible relapse of uveitis.

The invasion of ocular tissue by numerous OVA-specific $\mathrm{T}$ cells underlines the fact that activated $\mathrm{T}$ cells of any specificity can overcome the blood-retina barrier and enter the eye, but without causing destruction - unless they find their specific or cross-reactive antigen, and get reactivated. Staining for surface markers of T cell activation revealed that R14-specific GFP+ T cells are reactivated in the eye, but neither in lymphoid organs nor in blood, whereas OVA-specific GFP+ T cells experience no reactivation. To prove that antigen-specific reactivation is sufficient to induce uveitis, we injected OVA into the anterior chamber after the adoptive transfer of OVA-specific $\mathrm{T}$ cell lines. In this case we induced 'immunogenic uveitis' and also observed upregulation of activation markers on OVA-specific T cells isolated from the eye. Therefore, reactivated $\mathrm{T}$ cells can recruit inflammatory cells that cause destruction of ocular tissues.

\section{Differences between EAU Induced with S-Ag Peptide PDSAg or IRBP Peptide R14}

EAU in Lewis rats was regarded as an acute, monophasic disease, until Shao et al. [5] described relapsing disease after adoptive transfer of $\mathrm{T}$ cell lines specific for a peptide from IRBP (AA 1177-1191), which is an N-terminally truncated form of IRBP peptide R14.

We observed relapses of EAU after immunization of rats with peptide R14 in CFA and could reinduce EAU after prior disease induction with R14-CFA or adoptive transfer or R14-specific $\mathrm{T}$ cell lines but not after primary PDSAg-mediated disease [6]. T cell lines specific for R14 or PDSAg are both of the Th1 type; however, in culture supernatants of R14-specific T cells we find higher concentrations of cytokines than in supernatants of PDSAgspecific $\mathrm{T}$ cells, independent of the proliferation of the respective T cells [7].

R14- and PDSAg-mediated EAU is furthermore differently regulated with respect to chemokines. Disease 


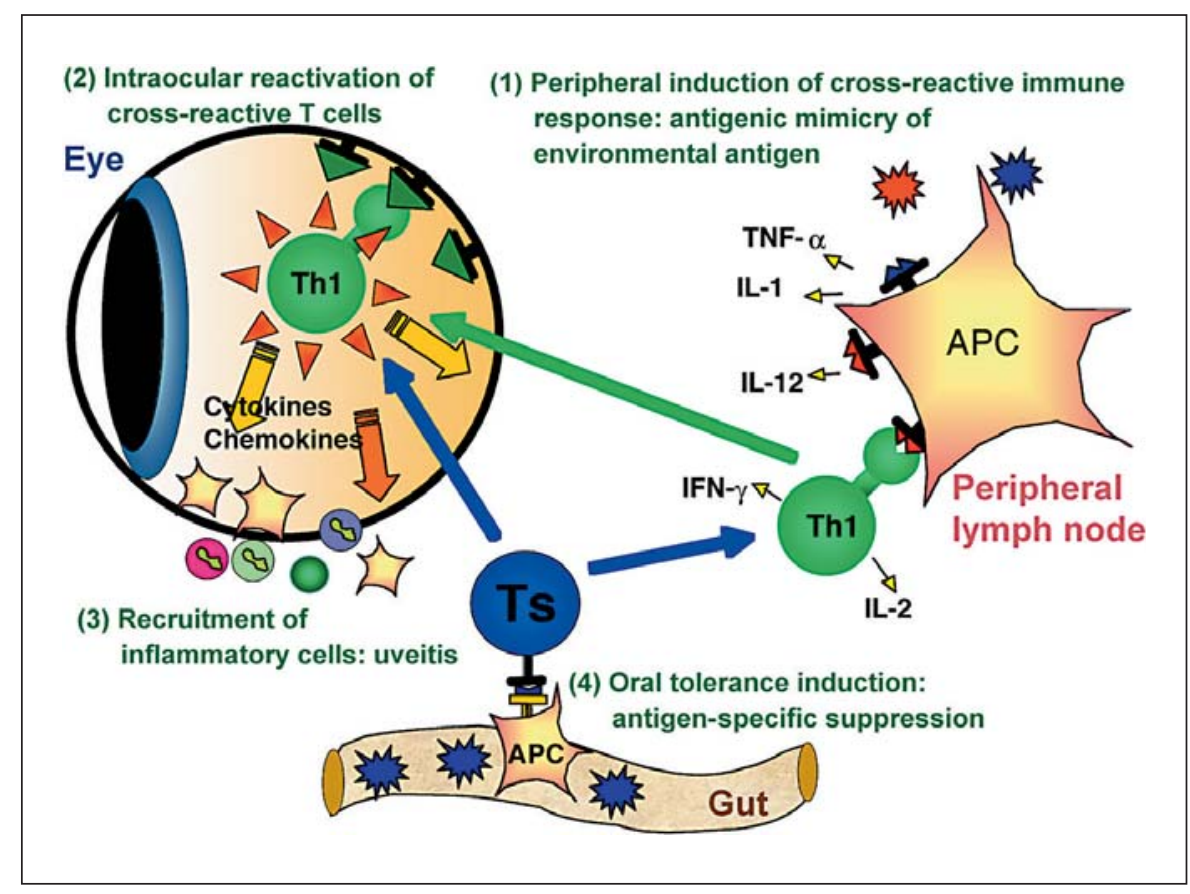

Fig. 1. Autoaggressive T cells in uveitis: peripheral induction, intraocular reactivation and therapeutic suppression by oral tolerance. (1) Naive T cells are activated outside of the eye (peripheral lymph nodes) by presentation of environmental antigens, which are cross-reactive with retinal autoantigen (e.g. bovine milk casein, rotavirus outer capsid protein). They become Th1 cells that secrete IL-2, IFN- $\gamma$ and TNF- $\alpha$. Once activated, these T cells are able to pass the blood-retina barrier and find a cross-reactive autoantigen within the eye. APC = Antigen-presenting cell. (2) Intraocular antigen contact leads to reactivation of the Th1 cells. (3) Subsequently, Th1 cells secrete chemokines and cytokines to attract inflammatory cells (rat EAU: mainly monocytes/macro-

induced by adoptive transfer of PDSAg-specific T cells was completely blocked by injection of N-terminally methionylated RANTES, a CCR1/CCR5 receptor antagonist, which had no effect on EAU induced by adoptive transfer of R14-specific T cells [7]. We could furthermore observe differences in the preferential site of infiltration of cells in R14- and PDSAg-induced diseases: while in PDSAg-mediated EAU cells mainly invade the retina from the choroid by destroying the retinal pigment epithelium, the cells in R14-mediated EAU immigrate mainly from the retinal vessels, destroying photoreceptors but not the retinal pigment epithelium.

Taken together, these data suggest that we have two antigen-dependent model diseases for uveitis in the same strain of rats.

Rat Models of Autoimmune Uveitis phages). (4) EAU can be suppressed by oral application of the retinal autoantigen/peptides used for experimental induction of disease. An HLA-class I B-derived peptide mimotope of S-Ag peptide PDSAg, B27PD, serves as oral tolerogen as well, whereas the mimicry peptides from rotavirus and bovine casein have no tolerogenic effect. The regulatory cells (Ts) are activated in the gut-associated lymphoid tissue (GALT) by the presentation of a respective tolerogen. They migrate in search of their antigen and of respective specific T effector cells outside the GALT, where they downregulate the activity of the Th1 effector cells in peripheral lymph nodes or probably also in the final target organ, the eye.

\section{Oral Tolerance Induction: Antigen-Specific Suppression as a Therapy of Uveitis}

Highly specific tolerance induction to only hit the autoaggressive immune response without impeding the general immune defense is a desired therapeutic approach for autoimmune diseases (fig. 1). Oral tolerance is a way to induce antigen-specific tolerance by feeding the respective (auto)antigen.

In the rat model, feeding of S-Ag or the peptide PDSAg can suppress S-Ag-induced EAU, and feeding IRBP suppresses IRBP-induced uveitis. We described a peptide derived from the sequence of HLA-B antigens that is called $\mathrm{B} 27 \mathrm{PD}$, although it is not only $\mathrm{B} 27$-specific. This peptide shares AA sequence homologies with peptide PDSAg and can serve as oral tolerogen to suppress EAU induced with 
S-Ag protein, peptide PDSAg and even IRBP protein [1]. Although the homology of IRBP and HLA peptide B27PD is not yet elucidated, data from equine uveitis also point to a cross-reactivity [8]. We could transfer protection from uveitis to naive rats by $\gamma \delta$ T cells isolated from the spleens of orally tolerized rats [9]. These $\gamma \delta \mathrm{T}$ cells proliferate in vitro in response to their respective antigen peptide or mimotope (PDSAg or B27PD), and $\alpha \beta$ T cells serve as antigen-presenting cells. The proliferation of these $\gamma \delta$ T cells is dependent on CD8 $\alpha \beta$ and MHC class II, as shown by the strong inhibitory effect of antibodies to CD8 $\beta$ and RT1.B, which is the restriction element of the tolerogenic peptides PDSAg and B27PD [10].

While we could induce severe EAU with peptides PDSAg, Cas and Rota, but not with B27PD, we were only able to induce tolerance to PDSAg-induced uveitis by feeding PDSAg or B27PD, but not using Rota or Cas, indicating that pathogenic antigens are not mandatorily tolerogenic and vice versa [10].

The high tolerogenicity accompanied with low pathogenicity makes peptide B27PD a highly interesting candidate for oral tolerance induction in patients.
We had therefore performed a therapeutic trial for patients with autoimmune uveitis [11], who were taking $4 \mathrm{mg}$ of encapsulated peptide B27PD 3 times a week for 12 weeks. All 8 patients could lower their corticosteroid doses either during or after oral peptide application with stable or increased visual acuity. During a 5-year followup 2 patients needed immunosuppressive therapy again (1 patient after 3 years, 2 after 4 and 5 years), but the average daily corticosteroid dose remained stable and significantly lower compared to the dose prior to oral peptide therapy.

An international multicenter trial with oral peptide B27PD for uveitis patients is in preparation.

Taken together, with the rat model leading to a new therapy for uveitis patients and the recent observations of relapsing as well as reinducible disease and the differences in regulation by chemokine receptors, we have a highly valuable model for human uveitis.

\section{References}

1 Wildner G, Thurau SR: Cross-reactivity between an HLA-B27-derived peptide and a retinal autoantigen peptide: a clue to major histocompatibility complex association with autoimmune disease. Eur J Immunol 1994; 24:2579-2585.

2 Sanui H, Redmond TM, Hu LH, Kuwabara T, Margalit H, Cornette JL, Wiggert B, Chader GJ, Gery I: Synthetic peptides derived from IRBP induce EAU and EAP in Lewis rats. Curr Eye Res 1988;7:727-735.

3 Wildner G, Diedrichs Mohring M: Autoimmune uveitis induced by molecular mimicry of peptides from rotavirus, bovine casein and retinal S-antigen. Eur J Immunol 2003; 33:2577-2587.
4 Thurau SR, Mempel TR, Flugel A, Diedrichs Mohring M, Krombach F, Kawakami N, Wildner G: The fate of autoreactive, GFP+ T cells in rat models of uveitis analyzed by intravital fluorescence microscopy and FACS. Int Immunol 2004;16:1573-1582.

5 Shao H, Shi H, Kaplan HJ, Sun D: Chronic recurrent autoimmune uveitis with progressive photoreceptor damage induced in rats by transfer of IRBP-specific T cells. J Neuroimmunol 2005;163:102-109.

6 Diedrichs-Möhring M, Hoffmann C, Wildner G: Antigen-dependent monophasic or recurrent autoimmune uveitis in rats. Int Immunol 2008;20:365-374.

7 Diedrichs Mohring M, Nelson PJ, Proudfoot AE, Thurau SR, Wildner G: The effect of the CC chemokine receptor antagonist MetRANTES on experimental autoimmune uveitis and oral tolerance. J Neuroimmunol $2005 ; 164: 22-30$.
8 Deeg CA, Thurau SR, Gerhards H, Ehrenhofer M, Wildner G, Kaspers B: Uveitis in horses induced by interphotoreceptor retinoid-binding protein is similar to the spontaneous disease. Eur J Immunol 2002;32: 2598-2606.

9 Wildner G, Hunig T, Thurau SR: Orally induced, peptide-specific gamma/delta TCR+ cells suppress experimental autoimmune uveitis. Eur J Immunol 1996;26:2140-2148.

10 Wildner G, Thurau SR, Diedrichs Mohring M: Gamma-delta T cells as orally induced suppressor cells in rats: in vitro characterization. Ann NY Acad Sci 2004;1029:416-421.

11 Thurau SR, Diedrichs Mohring M, Fricke H, Burchardi C, Wildner G: Oral tolerance with an HLA-peptide mimicking retinal autoantigen as a treatment of autoimmune uveitis. Immunol Lett 1999;68:205-212. 